TUM/T39-02-??

\title{
Single-particle potential in a chiral approach to nuclear matter including short range NN-terms
}

\author{
S. Fritsch and N. Kaiser \\ Physik Department T39, Technische Universität München, D-85747 Garching, Germany \\ email: nkaiser@physik.tu-muenchen.de
}

\begin{abstract}
We extend a recent chiral approach to nuclear matter of Lutz et al. [Phys. Lett. B474 (2000) 7] by calculating the underlying (complex-valued) single-particle potential $U\left(p, k_{f}\right)+i W\left(p, k_{f}\right)$. The potential for a nucleon at the bottom of the Fermi-sea, $U\left(0, k_{f 0}\right)=-20.0 \mathrm{MeV}$, comes out as much too weakly attractive in this approach. Even more seriously, the total single-particle energy does not rise monotonically with the nucleon momentum $p$, implying a negative effective nucleon mass at the Fermi-surface. Also, the imaginary single-particle potential, $W\left(0, k_{f 0}\right)=51.1 \mathrm{MeV}$, is too large. More realistic single-particle properties together with a good nuclear matter equation of state can be obtained if the short range contributions of non-pionic origin are treated in mean-field approximation (i.e. if they are not further iterated with $1 \pi$-exchange). We also consider the equation of state of pure neutron matter $\bar{E}_{n}\left(k_{n}\right)$ and the asymmetry energy $A\left(k_{f}\right)$ in that approach. The downward bending of these quantities above nuclear matter saturation density seems to be a generic feature of perturbative chiral pion-nucleon dynamics.
\end{abstract}

PACS: 12.38.Bx, 21.65.+f

Keywords: Nuclear matter equation of state; Complex single-particle potential in symmetric nuclear matter; Neutron matter; Asymmetry energy

\section{Introduction}

The present status of the nuclear matter problem is that a quantitatively successful description can be achieved, using advanced many-body techniques [1], in a non-relativistic framework when invoking an adjustable three-body force. Alternative relativistic mean-field approaches, including non-linear terms with adjustable parameters or explicitly density-dependent point couplings, are also widely used for the calculation of nuclear matter properties and finite nuclei [2].

In recent years a novel approach to the nuclear matter problem based on effective field theory (in particular chiral perturbation theory) has emerged [3, 4, 5]. The key element there is a separation of long- and short-distance dynamics and an ordering scheme in powers of small momenta. At nuclear matter saturation density the Fermi-momentum $k_{f 0}$ and the pion mass $m_{\pi}$ are comparable scales $\left(k_{f 0} \simeq 2 m_{\pi}\right)$, and therefore pions must be included as explicit degrees of freedom in the description of the nuclear many-body dynamics. The contributions to the energy

\footnotetext{
${ }^{1}$ Work supported in part by BMBF, GSI and DFG.
} 
per particle of isospin-symmetric nuclear matter $\bar{E}\left(k_{f}\right)$ originating from chiral pion-nucleon dynamics have been calculated up to three-loop order in refs. 3, 4. Both calculations include the $1 \pi$-exchange Fock-diagram and the iterated $1 \pi$-exchange Hartree- and Fock-diagrams. In ref. [4] irreducible $2 \pi$-exchange is also taken into account and a momentum cut-off $\Lambda$ is used to regularize the few divergent parts associated with chiral $2 \pi$-exchange. The resulting cut-off dependent contribution to $\bar{E}\left(k_{f}\right)$ is completely equivalent to that of a zero-range NN-contact interaction (see eq.(15) in ref.[4]). At that point the work of Lutz et al. [3] deviates and it follows a different strategy. Two zero-range NN-contact interactions (acting in ${ }^{3} S_{1}$ and ${ }^{1} S_{0}$ NN-states) proportional to the parameters $g_{0}+g_{A}^{2} / 4$ and $g_{1}+g_{A}^{2} / 4$ are introduced (see eq.(4) in ref.[3]). The components proportional to $g_{A}^{2} / 4$ cancel the zero-range contribution generated by the $1 \pi$-exchange Fock-diagram. The other components proportional to $g_{0}$ and $g_{1}$ are understood to subsume all non-perturbative short-range NN-dynamics relevant at densities around nuclear matter saturation density $\rho_{0}$. In order to be consistent with this interpretation the NN-contact vertices proportional to $g_{0,1}$ are allowed to occur only in first order. Furthermore, according to ref.[6] pions can be treated perturbatively (at least) in the ${ }^{1} S_{0}$ partial-wave of NN-scattering if the zero-range pieces they generate are removed order by order. Therefore, the NN-contact vertex proportional to $g_{A}^{2} / 4$ occurs also in higher orders (see Fig.1 in ref. 33 which includes diagrams with "filled circle" and "open circle" vertices).

Despite their differences in the treatment of the effective short-range NN-dynamics both approaches [3, 14] are able to reproduce correctly the empirical nuclear matter properties (saturation density $\rho_{0}$, binding energy per particle $-\bar{E}\left(k_{f 0}\right)$ and compressibility $K$ ) by adjusting only one parameter, either the coupling $g_{0}+g_{1} \simeq 3.23$ or the cut-off $\Lambda \simeq 0.65 \mathrm{GeV}$. Note that in dimensional regularization all diagrams evaluated in ref. 3. are finite. In the chiral approach of the Munich group [4, 5] the asymmetry energy $A\left(k_{f}\right)$, the energy per particle of pure neutron matter $\bar{E}_{n}\left(k_{n}\right)$ as well as the (complex) single-particle potential $U\left(p, k_{f}\right)+i W\left(p, k_{f}\right)$ below the Fermi-surface $\left(p \leq k_{f}\right)$ have been calculated. Good results (in particular for the asymmetry energy, $A\left(k_{f 0}\right)=33.8 \mathrm{MeV}$, and the depth of the single-particle potential, $\left.U\left(0, k_{f 0}\right)=-53.2 \mathrm{MeV}\right)$ have been obtained with the single cut-off scale $\Lambda \simeq 0.65 \mathrm{GeV}$ adjusted to the binding energy per particle $-\bar{E}\left(k_{f 0}\right)=15.3 \mathrm{MeV}$. Moreover, when extended to finite temperatures [7] this approach reproduces the liquid-gas phase transition of isospin-symmetric nuclear, however, with a too high value of the critical temperature $T_{c}=25.5 \mathrm{MeV}$.

It is the purpose of this work to investigate in the approach of Lutz et al. [3] the single-particle potential $U\left(p, k_{f}\right)+i W\left(p, k_{f}\right)$ in isospin-symmetric nuclear matter as well as the neutron matter

equation of state $\bar{E}_{n}\left(k_{n}\right)$ and the asymmetry energy $A\left(k_{f}\right)$. One of our major conclusions will be that any strong short-range NN-dynamics of non-pionic origin should be kept at the meanfield level. It should not be further iterated with pion-exchange in contrast to the prescription of power-counting rules.

\section{Nuclear matter equation of state}

Let us first reconsider the equation of state of isospin-symmetric nuclear matter as it follows from the calculation of ref. [3]. Even though all contributions to the energy per particle $\bar{E}\left(k_{f}\right)$ have been given explicitly in ref. [3] we prefer to write down again the extra terms generated by the NN-contact interactions proportional to $g_{0,1}+g_{A}^{2} / 4$ (using a more compact notation). The first diagram in Fig. 1 gives rise to a contribution to energy per particle of the form:

$$
\bar{E}\left(k_{f}\right)=-\frac{(\gamma+1) g_{A}^{2} k_{f}^{3}}{\left(4 \pi f_{\pi}\right)^{2}},
$$



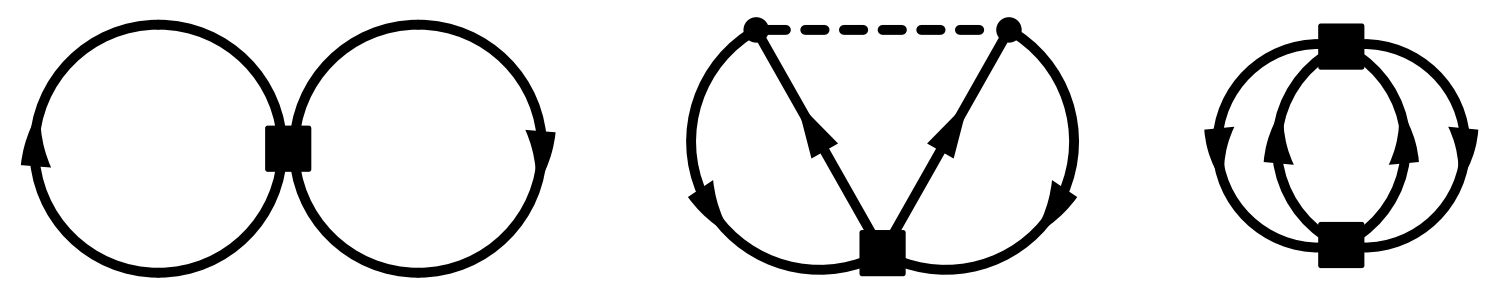

Figure 1: Additional in-medium diagrams generated by the NN-contact interactions introduced in ref.[3]. The two NN-contact interactions proportional to $\gamma+1$ and $\gamma_{n}+1$ are symbolized by the filled square vertex. The last diagram is to be understood such that quadratic terms (such as $\gamma^{2}, \gamma \gamma_{n}$ and $\gamma_{n}^{2}$ ) are omitted.

where we have introduced (for notational convenience) the coefficient $\gamma$ by the relation $(\gamma+$ 1) $g_{A}^{2} / 2=g_{0}+g_{1}+g_{A}^{2} / 2$. In the second and third diagram in Fig. 1 the contact-interaction proportional to $\gamma+1$ is iterated with $1 \pi$-exchange or with itself (dropping the $\gamma^{2}$-contribution). Putting a medium-insertion? at each of two nucleon propagators with equal orientation one gets:

$$
\bar{E}\left(k_{f}\right)=\frac{3(\gamma+1) g_{A}^{4} M m_{\pi}^{4}}{5(8 \pi)^{3} f_{\pi}^{4}}\left[11 u-\frac{1}{2 u}-\left(10+8 u^{2}\right) \arctan 2 u+\left(\frac{1}{8 u^{3}}+\frac{5}{2 u}\right) \ln \left(1+4 u^{2}\right)\right],
$$

with the abbreviation $u=k_{f} / m_{\pi}$. One observes that eq.(2) receives no contribution from the third diagram in Fig. 1 since $\int_{0}^{\infty} d l 1$ is set to zero in dimensional regularization. The second and third diagram in Fig. 1 with three medium-insertions give rise to the following contribution to the energy per particle:

$$
\bar{E}\left(k_{f}\right)=\frac{9 g_{A}^{4} M m_{\pi}^{4}}{\left(4 \pi f_{\pi}\right)^{4} u^{3}} \int_{0}^{u} d x x^{2} \int_{-1}^{1} d y\left[2 u x y+\left(u^{2}-x^{2} y^{2}\right) H\right]\left[\frac{\gamma+1}{2} \ln \left(1+s^{2}\right)-\frac{s^{2}}{4}\right],
$$

with the auxiliary functions $H=\ln (u+x y)-\ln (u-x y)$ and $s=x y+\sqrt{u^{2}-x^{2}+x^{2} y^{2}}$. In the chiral limit $m_{\pi}=0$ only the contribution coming from the last term, $-s^{2} / 4$, in the second square bracket survives. The corresponding double integral $\int_{0}^{u} d x x^{2} \int_{-1}^{1} d y \ldots$ has the value $2 u^{7}(\ln 4-11) / 105$. The expansion of the energy per particle up to order $\mathcal{O}\left(k_{f}^{4}\right)$ is completed by adding to the terms eqs. $(1,2,3)$ the contributions from the (relativistically improved) kinetic energy, from $1 \pi$-exchange and from iterated $1 \pi$-exchange written down in eqs.(5-11) of ref. 四. In case of the $1 \pi$-exchange contribution (eq.(6) in ref.[[]]) we neglect of course the small relativistic $1 / M^{2}$-correction of order $\mathcal{O}\left(k_{f}^{5}\right)$.

Now, we have to fix parameters. The pion decay constant $f_{\pi}=92.4 \mathrm{MeV}$ and the nucleon mass $M=939 \mathrm{MeV}$ are well-known. As in ref. [4] we choose the value $g_{A}=1.3$. This corresponds via the Goldberger-Treiman relation to a $\pi N N$-coupling constant of $g_{\pi N}=g_{A} M / f_{\pi}=$ 13.2 which agrees with present empirical determinations of $g_{\pi N}$ from $\pi N$-dispersion relation analyses [8]. We set $m_{\pi}=135 \mathrm{MeV}$ (the neutral pion mass) since this is closest to the expected value of the pion mass in the absence of isospin-breaking and electromagnetic effects.

The dashed line in Fig. 2 shows the equation of state of isospin-symmetric nuclear matter in the approach of ref. [3] using the abovementioned input parameters. The coefficient $\gamma=4.086$ has been adjusted such that the minimum of the saturation curve lies at $\bar{E}\left(k_{f 0}\right)=-15.3 \mathrm{MeV}$ [9]. The predicted equilibrium density $\rho_{0}=0.138 \mathrm{fm}^{-3}$ (corresponding to a Fermi-momentum of

\footnotetext{
${ }^{2}$ This is a technical notation for the difference between the in-medium and vacuum nucleon propagator. For further details, see section 2 in ref. [4].
} 


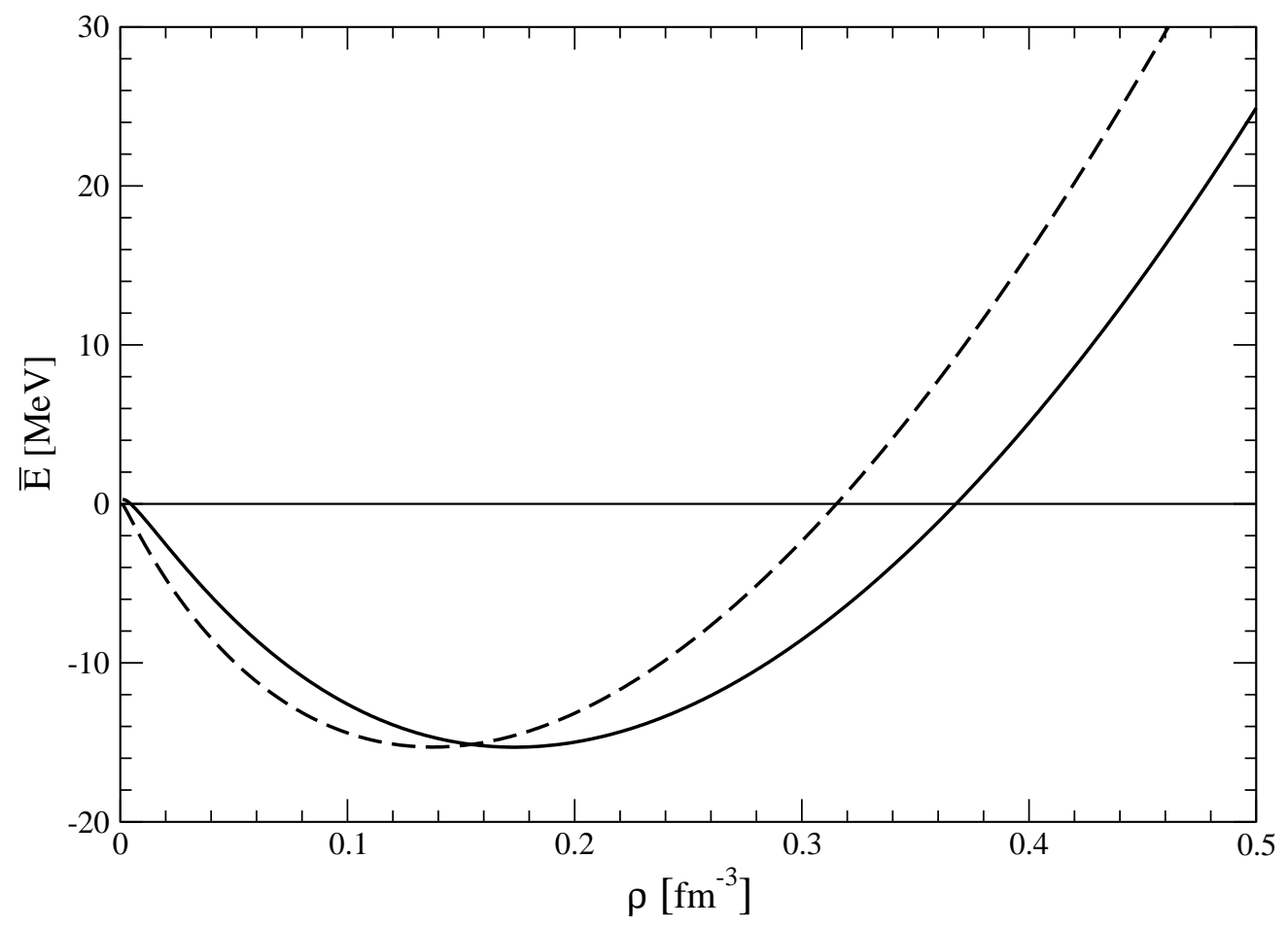

Figure 2: The energy per particle of isospin-symmetric nuclear matter $\bar{E}\left(k_{f}\right)$ versus the nucleon density $\rho=2 k_{f}^{3} / 3 \pi^{2}$. The dashed line corresponds to the approach of ref. [3]. The full line results if the NN-contact interaction is treated in mean-field approximation. In each case the coefficient $\gamma$ is adjusted such that the saturation minimum lies at $\bar{E}\left(k_{f 0}\right)=-15.3 \mathrm{MeV}$.

$\left.k_{f 0}=250.1 \mathrm{MeV}\right)$ is somewhat too low. The same holds for the nuclear matter compressibility $K=k_{f 0}^{2} \bar{E}^{\prime \prime}\left(k_{f 0}\right)=202 \mathrm{MeV}$. Of course, if we use the input parameters of ref. [3] $\left(f_{\pi}=93 \mathrm{MeV}\right.$, $g_{A}=1.26, m_{\pi}=140 \mathrm{MeV}$ and $g_{0}+g_{1}=3.23$ corresponding to $\left.\gamma=4.07\right)$ we exactly reproduce the numerical results of that work. We emphasize that the different treatment of the two components of the NN-contact interaction is essential in order to get (realistic) nuclear binding and saturation in the framework of ref. [3]. If both components were treated on equal footing in first order (technically this is realized by deleting the contribution coming from the third diagram in Fig. 1) the energy per particle $\bar{E}\left(k_{f}\right)$ would not even develop a minimum.

\section{Real single-particle potential}

Next, we turn to the real part of the single-particle potential $U\left(p, k_{f}\right)$ below the Fermi-surface $\left(p \leq k_{f}\right)$ in the framework of ref.[3]. As outlined in ref. [5] the contributions to $U\left(p, k_{f}\right)$ can be classified as two-body and three-body potentials. From the first diagram in Fig. 1 one gets a contribution to the two-body potential of the form:

$$
U_{2}\left(p, k_{f}\right)=-\frac{2(\gamma+1) g_{A}^{2} k_{f}^{3}}{\left(4 \pi f_{\pi}\right)^{2}}
$$




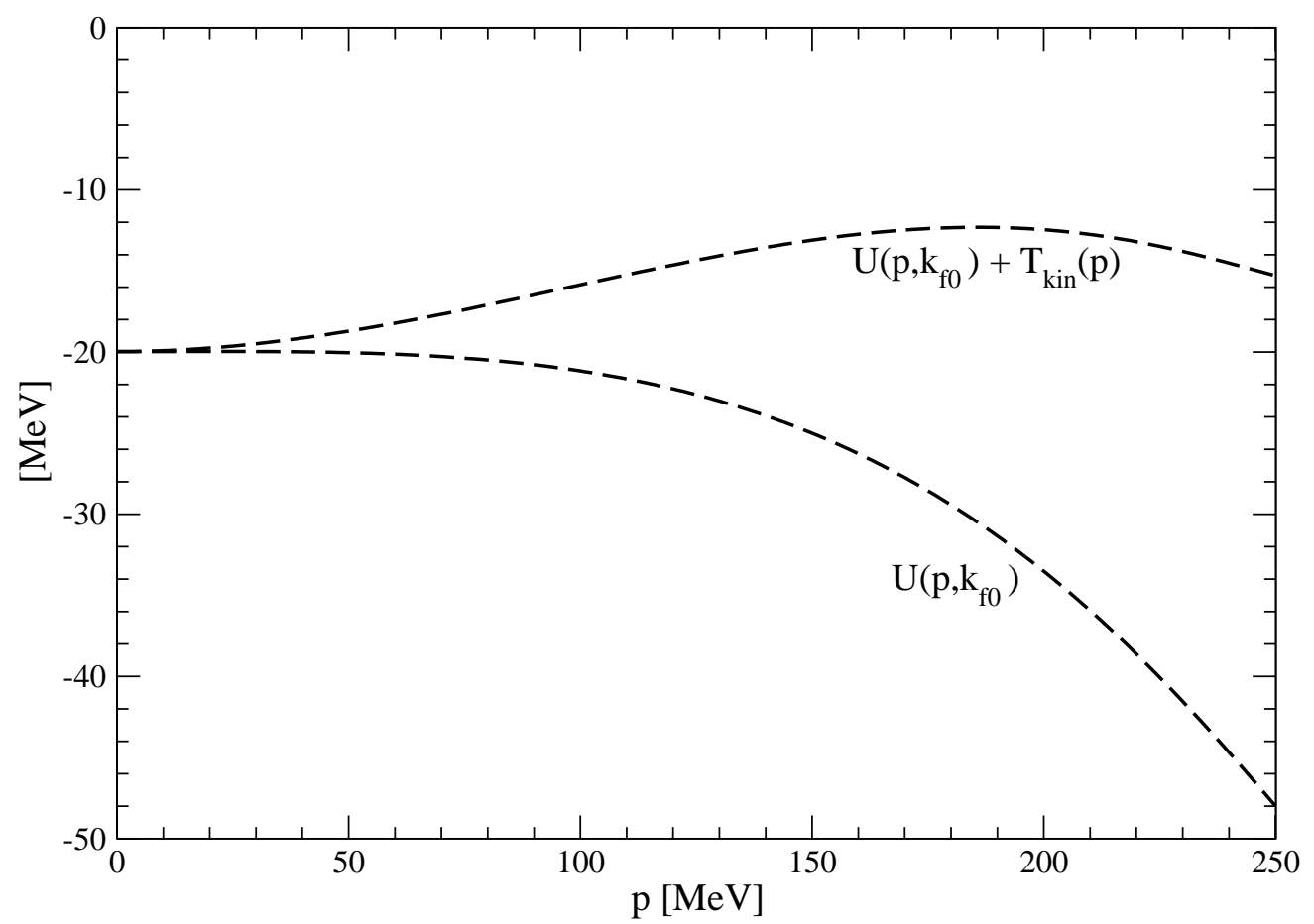

Figure 3: The lower curve shows the real part of the single-particle potential $U\left(p, k_{f 0}\right)$ at saturation density $k_{f 0}=250.1 \mathrm{MeV}$ in the approach of Lutz et al. [3]. The upper curve includes in addition the relativistically improved kinetic energy $T_{\text {kin }}(p)=p^{2} / 2 M-p^{4} / 8 M^{3}$.

which is just twice its contribution to the energy per particle (see eq.(1)). From the second diagram in Fig. 1 one derives a contribution to the two-body potential of the form:

$$
\begin{aligned}
U_{2}\left(p, k_{f}\right)= & \frac{(\gamma+1) g_{A}^{4} M m_{\pi}^{4}}{(4 \pi)^{3} f_{\pi}^{4}}\left\{u+\frac{1}{4 x}\left(x^{3}-3 x-3 u^{2} x-2 u^{3}\right) \arctan (u+x)\right. \\
& +\frac{1}{4 x}\left(x^{3}-3 x-3 u^{2} x+2 u^{3}\right) \arctan (u-x) \\
& \left.+\frac{1}{8 x}\left(1+3 u^{2}-3 x^{2}\right) \ln \frac{1+(u+x)^{2}}{1+(u-x)^{2}}\right\},
\end{aligned}
$$

with the abbreviation $x=p / m_{\pi}$. The second and third diagram in Fig. 1 give each rise to three different contributions to the three-body potential. Altogether, they read:

$$
\begin{aligned}
U_{3}\left(p, k_{f}\right)= & \frac{3 g_{A}^{4} M m_{\pi}^{4}}{\left(4 \pi f_{\pi}\right)^{4}} \int_{-1}^{1} d y\left\{\left[2 u x y+\left(u^{2}-x^{2} y^{2}\right) H\right]\left[\frac{\gamma+1}{2} \ln \left(1+s^{2}\right)-\frac{s^{2}}{4}\right]\right. \\
& +\int_{-x y}^{s-x y} d \xi\left[2 u \xi+\left(u^{2}-\xi^{2}\right) \ln \frac{u+\xi}{u-\xi}\right] \frac{(2 \gamma+1)(x y+\xi)-(x y+\xi)^{3}}{2\left[1+(x y+\xi)^{2}\right]} \\
& \left.+\int_{0}^{u} d \xi \frac{\xi^{2}}{x}\left[(\gamma+1) \ln \left(1+\sigma^{2}\right)-\frac{\sigma^{2}}{2}\right] \ln \frac{|x+\xi y|}{|x-\xi y|}\right\},
\end{aligned}
$$

with the auxiliary function $\sigma=\xi y+\sqrt{u^{2}-\xi^{2}+\xi^{2} y^{2}}$. The real single-particle potential $U\left(p, k_{f}\right)$ is completed by adding to the terms eqs. $(4,5,6)$ the contributions from $1 \pi$-exchange and iterated $1 \pi$-exchange written down in eqs.(8-13) of ref. [5]. Again, the (higher order) relativistic $1 / M^{2}$ correction to $1 \pi$-exchange (see eq.(8) in ref. [5]) is neglected for reasons of consistency. 


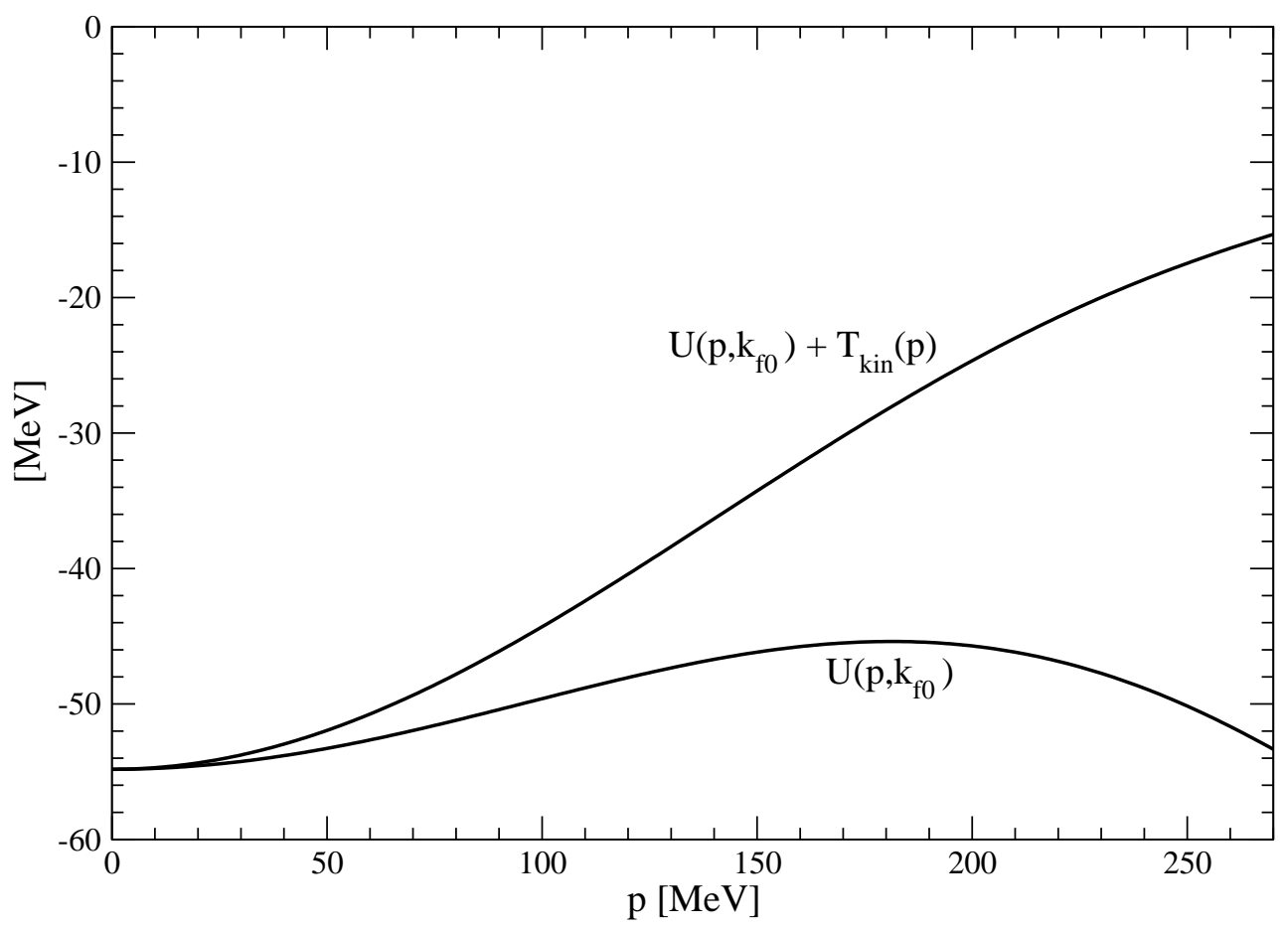

Figure 4: The lower curve shows the real part of the single-particle potential $U\left(p, k_{f 0}\right)$ at saturation density $k_{f 0}=270.3 \mathrm{MeV}$ in a mean-field treatment of the NN-contact interaction.

The lower curve in Fig. 3 shows the momentum dependence of the real single-particle potential $U\left(p, k_{f 0}\right)$ at saturation density $k_{f 0}=250.1 \mathrm{MeV}$ as it arises in the framework of Lutz et al. [3]. The predicted potential depth for a nucleon at the bottom of the Fermi-sea is only $U\left(0, k_{f 0}\right)=-20.0 \mathrm{MeV}$. In magnitude this is much smaller than the typical depth $U_{0} \simeq-53 \mathrm{MeV}$ of the empirical optical model potential [10] or the nuclear shell model potential [11. The upper curve in Fig. 3 includes the (relativistically improved) single-nucleon kinetic energy $T_{\text {kin }}(p)=p^{2} / 2 M-p^{4} / 8 M^{3}$. As required by the Hugenholtz-van-Hove theorem 12] this curve ends at the Fermi-surface $p=k_{f 0}$ with the value $\bar{E}\left(k_{f 0}\right)=-15.3 \mathrm{MeV}$. A further important check is provided by the sum rule for the two- and three-body potentials $U_{2,3}\left(p, k_{f}\right)$ written down in eq.(5) of ref. [5]. It holds with very high numerical accuracy in the present calculation.

The momentum dependence of the two (dashed) curves in Fig. 3 is completely unrealistic. Most seriously, the total single-particle energy $T_{\text {kin }}(p)+U\left(p, k_{f 0}\right)$ (upper curve) does not rise monotonically with the nucleon momentum $p$, but instead it starts to bend downward above $p \simeq 190 \mathrm{MeV}$. This implies a negative effective nucleon mass at the Fermi-surface, $M^{*}\left(k_{f 0}\right) \simeq$ $-3.5 M$, and a negative density of states with dramatic consequences for the finite temperature behavior of nuclear matter. Because of such pathological features of the underlying singleparticle potential the scheme of Lutz et al. [3] has to be rejected in its present form.

The overly strong momentum dependence of $U\left(p, k_{f 0}\right)$ comes from the second and third diagram in Fig. 1 in which the NN-contact interaction proportional to the large coefficient $\gamma+1$ is further iterated. We propose to drop these three-loop diagrams and to keep the NN-contact interaction (of unspecified dynamical origin) at the mean-field level. The resulting equation of state obtained by leaving out the contributions eqs. $(2,3)$ and adjusting $\gamma=6.198$ is shown by the full line in Fig. 2. The predicted saturation density is now $\rho_{0}=0.174 \mathrm{fm}^{-3}$ (corresponding to a Fermi-momentum of $k_{f 0}=270.3 \mathrm{MeV}$ ) and the nuclear matter compressibility has the 
value $K=253 \mathrm{MeV}$. Note that the scheme of ref.[3] modified by a mean-field treatment of the $\mathrm{NN}$-contact interaction becomes equivalent to the truncation at fourth order in small momenta of our previous work [4, 5] after the identification of parameters, $\gamma+1=10 g_{A}^{2} \Lambda M /\left(4 \pi f_{\pi}\right)^{2}$, with $\Lambda$ denoting the cut-off scale.

The lower full curve in Fig. 4 shows the momentum dependence of the real single-particle potential at saturation density $k_{f 0}=270.3 \mathrm{MeV}$ which results in a mean-field approximation of the NN-contact interaction (by leaving out the contributions eqs. $(5,6)$ ). The predicted potential depth $U\left(0, k_{f 0}\right)=-54.8 \mathrm{MeV}$ is in good agreement with that of optical model [10] or nuclear shell model potentials [11. Most importantly, the total single-particle energy $T_{\text {kin }}(p)+U\left(p, k_{f 0}\right)$ (upper curve) grows now monotonically with the nucleon momentum $p$, as it should. The upand downward bending of the lower full curve in Fig. 4 is however still too strong. The negative slope of $U\left(p, k_{f 0}\right)$ at the Fermi-surface $p=k_{f 0}$ leads to a too large effective nucleon mass $M^{*}\left(k_{f 0}\right) \simeq 2.9 \mathrm{M}$ which reflects itself in a too high critical temperature $T_{c} \simeq 25 \mathrm{MeV}$ of the liquid-gas phase transition [7]. More elaborate calculation of nuclear matter in effective (chiral) field theory are necessary in order to cure this problem of the too large effective nucleon mass $M^{*}\left(k_{f 0}\right)$.

\section{Imaginary single-particle potential}

In this section, we discuss the imaginary part of the single-particle potential $W\left(p, k_{f}\right)$ for $p \leq k_{f}$ as it arises in the scheme of Lutz et al. [3]. This quantity determines the half-width of nucleonhole states in the Fermi-sea. As outlined in ref. [5] the contributions to $W\left(p, k_{f}\right)$ can be classified as two-body, three-body and four-body terms. From the second and third diagrams in Fig. 1 one derives a two-body term of the form:

$$
\begin{aligned}
W_{2}\left(p, k_{f}\right)= & \frac{g_{A}^{4} M m_{\pi}^{4}}{(8 \pi)^{3} f_{\pi}^{4}}\left\{u^{2} x^{2}+\frac{3 u^{4}}{2}-\frac{x^{4}}{10}+(\gamma+1)\left[4+14 u^{2}-\frac{22 x^{2}}{3}\right.\right. \\
& +\frac{2}{x}\left(3 x^{2}-3 u^{2}-1\right)[\arctan (u+x)-\arctan (u-x)] \\
& +\frac{1}{x}\left(x^{3}-3 x-3 u^{2} x-2 u^{3}\right) \ln \left[1+(u+x)^{2}\right] \\
& \left.\left.+\frac{1}{x}\left(x^{3}-3 x-3 u^{2} x+2 u^{3}\right) \ln \left[1+(u-x)^{2}\right]\right]\right\} .
\end{aligned}
$$

The associated three-body term reads:

$$
\begin{aligned}
W_{3}\left(p, k_{f}\right)= & \frac{3 \pi g_{A}^{4} M m_{\pi}^{4}}{\left(4 \pi f_{\pi}\right)^{4}} \int_{-1}^{1} d y\left\{( \gamma + 1 ) \left[2 x y(s-\arctan s)-\frac{s^{2}}{2}\right.\right. \\
& \left.+\left(\frac{1}{2}+u^{2}-x^{2} y^{2}\right) \ln \left(1+s^{2}\right)\right]+s^{2}\left(\frac{s^{2}}{8}-\frac{u^{2}}{2}-\frac{s x y}{3}+\frac{x^{2} y^{2}}{2}\right) \\
& \left.+\int_{0}^{u} d \xi \frac{\xi^{2}}{x} \theta(x-\xi|y|)\left[(\gamma+1) \ln \left(1+\sigma^{2}\right)-\frac{\sigma^{2}}{2}\right]\right\},
\end{aligned}
$$

and the four-body term is given by the expression:

$$
\begin{aligned}
W_{4}\left(p, k_{f}\right)= & \frac{3 \pi g_{A}^{4} M m_{\pi}^{4}}{\left(4 \pi f_{\pi}\right)^{4}}\left\{(\gamma+1)\left[\frac{4 x^{2}}{3}-1+\ln \left(1+4 x^{2}\right)+\left(\frac{1}{2 x}-2 x\right) \arctan 2 x\right]\right. \\
& -\frac{4 x^{4}}{15}+\int_{-1}^{1} d y\left[s^{2}\left(\frac{u^{2}}{2}-\frac{s^{2}}{4}+\frac{2 s x y}{3}-\frac{x^{2} y^{2}}{2}\right)\right. \\
& \left.\left.+(\gamma+1)\left[4 x y(\arctan s-s)+s^{2}+\left(x^{2} y^{2}-1-u^{2}\right) \ln \left(1+s^{2}\right)\right]\right]\right\} .
\end{aligned}
$$




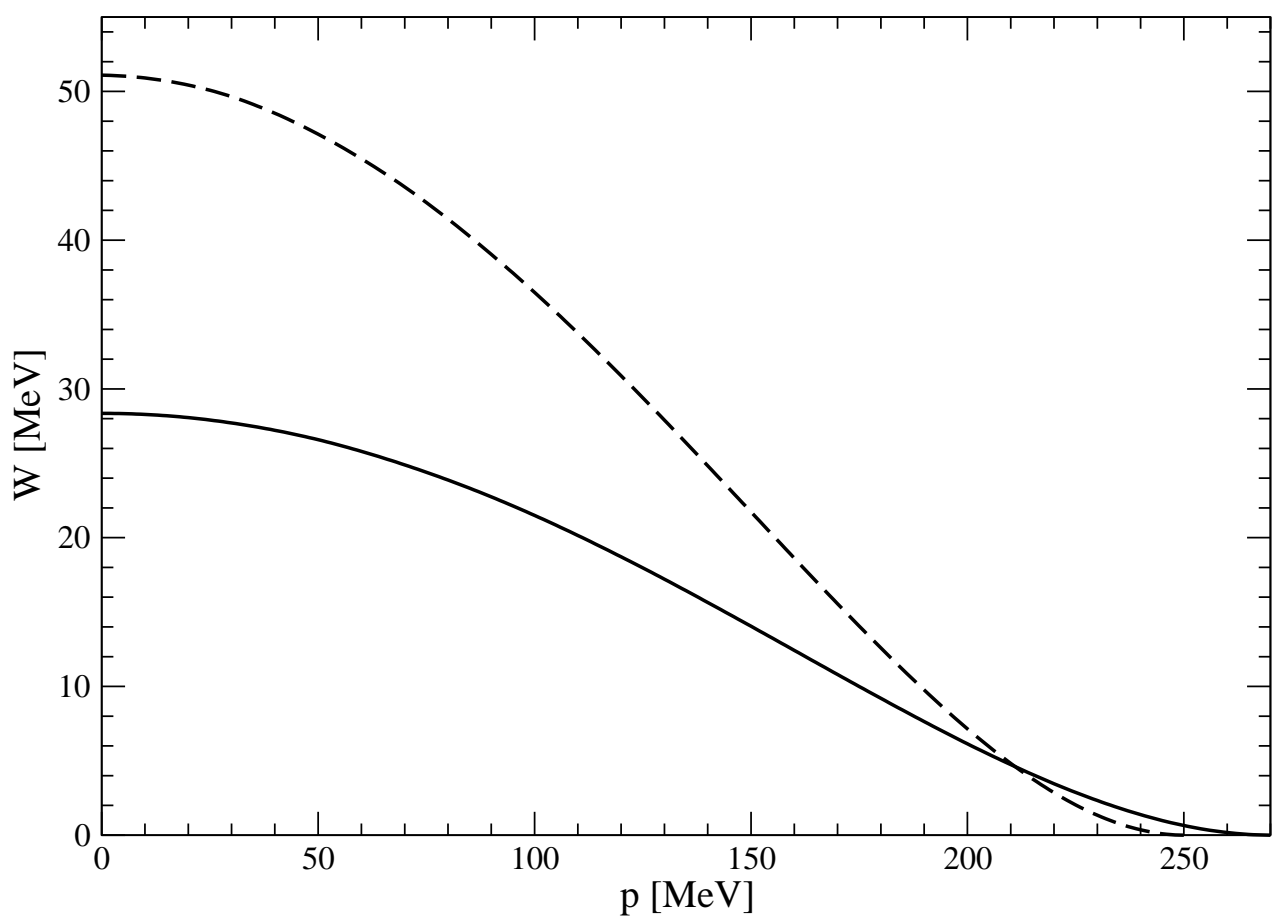

Figure 5: The imaginary part of the single-particle potential $W\left(p, k_{f 0}\right)$ at saturation density versus the nucleon momentum $p$. The dashed line corresponds to the approach of Lutz et al. [3] and the full line shows the result obtained in a mean-field treatment of the NN-contact interaction.

The additional contributions from the iterated 1 $1 \pi$-exchange Hartree- and Fock-diagram are collected in eqs.(20-25) of ref. [5]. The total imaginary single-particle potential evaluated at zero nucleon momentum $(p=0)$ can even be written as a closed form expression:

$$
\begin{aligned}
W\left(0, k_{f}\right)= & \frac{3 \pi g_{A}^{4} M m_{\pi}^{4}}{\left(4 \pi f_{\pi}\right)^{4}}\left\{\frac{u^{4}}{2}+(\gamma-2) u^{2}-\frac{2 u^{2}}{1+u^{2}}+\frac{\pi^{2}}{12}+\operatorname{Li}_{2}\left(-1-u^{2}\right)\right. \\
& \left.+\left[4-\gamma+\ln \left(2+u^{2}\right)-\frac{1}{2} \ln \left(1+u^{2}\right)\right] \ln \left(1+u^{2}\right)\right\},
\end{aligned}
$$

where $\operatorname{Li}_{2}\left(-a^{-1}\right)=\int_{0}^{1} d \zeta(\zeta+a)^{-1} \ln \zeta$ denotes the conventional dilogarithmic function.

The dashed line in Fig. 5 shows the momentum dependence of the imaginary single-particle potential $W\left(p, k_{f 0}\right)$ at saturation density $k_{f 0}=250.1 \mathrm{MeV}$ as it arises in the approach of ref. [3]. The predicted value $W\left(0, k_{f 0}\right)=51.1 \mathrm{MeV}$ lies outside the range $20-40 \mathrm{MeV}$ obtained in calculations based on (semi)-realistic NN-forces [13, 14]. The full line in Fig. 5 corresponds to a mean-field approximation of the NN-contact interaction. Up to a slight change in the equilibrium Fermi-momentum $k_{f 0}=270.3 \mathrm{MeV}$ the full curve in Fig. 5 agrees with the one shown in Fig. 4 of ref. [5]. The considerably reduced value $W\left(0, k_{f 0}\right)=28.4 \mathrm{MeV}$ indicates the large contribution of the iterated diagrams in Fig. 1 to the imaginary single-particle potential $W\left(p, k_{f}\right)$. Note that both curves in Fig. 5 vanish quadratically near the Fermi-surface as required by Luttinger's theorem [15]. As further check on our calculation we verified the zero sum rule: $\int_{0}^{k_{f}} d p p^{2}\left[6 W_{2}\left(p, k_{f}\right)+4 W_{3}\left(p, k_{f}\right)+3 W_{4}\left(p, k_{f}\right)\right]=0$, for the two-, three- and four-body components $W_{2,3,4}\left(p, k_{f}\right)$ written in eqs. $(7,8,9)$. 


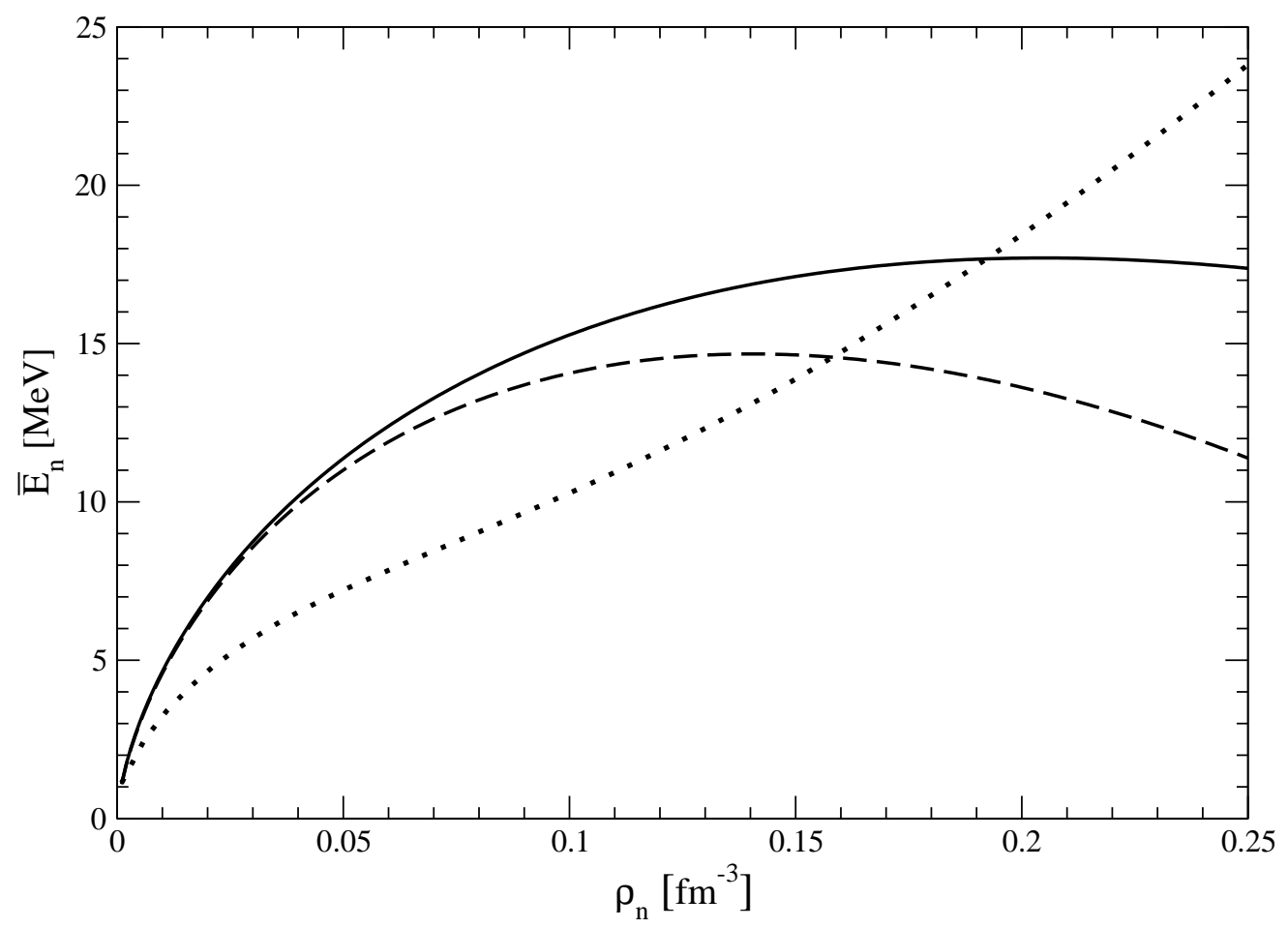

Figure 6: The energy per particle of pure neutron matter $\bar{E}_{n}\left(k_{n}\right)$ versus the neutron density $\rho_{n}=k_{n}^{3} / 3 \pi^{2}$. The dashed line corresponds to the approach of Lutz et al. [3] and the full line shows the result obtained in mean-field approximation of the $n n$-contact interaction. The dotted line stems from the many-body calculation of the Urbana group [16].

\section{Neutron matter}

In this section we discuss the equation of state of pure neutron matter. In the scheme of Lutz et al. [3] the energy per particle of pure neutron matter $\bar{E}_{n}\left(k_{n}\right)$ depends exclusively on the coefficient $g_{1}$ parameterizing the short-range NN-interaction in the channel with total isospin $I=1$. There is no need to write down explicitly the contributions of the diagrams in Fig. 1 to $\bar{E}_{n}\left(k_{n}\right)$. These expressions are easily obtained from eqs. $(1,2,3)$ by replacing $k_{f}$ by the neutron Fermi-momentum $k_{n}$, by replacing the coefficient $\gamma$ by a new one $\gamma_{n}$, and by multiplying the formulas with a relative isospin factor $1 / 3$. The relation $\left(\gamma_{n}+1\right) g_{A}^{2} / 4=g_{1}+g_{A}^{2} / 4$ defines this new coefficient $\gamma_{n}$. The additional contributions to $\bar{E}_{n}\left(k_{n}\right)$ from the kinetic energy, $1 \pi$ exchange and iterated $1 \pi$-exchange are written down in eqs.(32-37) of ref.[四] (neglecting again the relativistic $1 / M^{2}$-correction to $1 \pi$-exchange).

The dashed line in Fig. 6 shows the energy per particle of pure neutron matter $\bar{E}_{n}\left(k_{n}\right)$ versus the neutron density $\rho_{n}=k_{n}^{3} / 3 \pi^{2}$ as it arises in the approach of ref.[3]. The coefficient $\gamma_{n}=0.055$ has been adjusted to the empirical value of the asymmetry energy $A\left(k_{f 0}=250.1 \mathrm{MeV}\right)=$ 33.2 $\mathrm{MeV}$ (see next section). The downward bending of the dashed curve in Fig. 6 above $\rho_{n}>$ $0.15 \mathrm{fm}^{-3}$ is even stronger than in our previous work [4] (see Fig. 8 therein). This property can be understood by taking the chiral limit $\left(m_{\pi} \rightarrow 0\right)$ of the calculated neutron matter equation of state and considering the coefficient $\beta_{n}$ in front of the term $k_{n}^{4} / M^{3}$. In the approach of Lutz et al. [3] one has:

$$
\beta_{n}=-\frac{1}{70}\left(\frac{g_{\pi N}}{4 \pi}\right)^{4}\left(4 \pi^{2}+17+16 \ln 2\right)-\frac{3}{56}=-1.23,
$$

which is 2.2 times the negative value of $\beta_{n}$ found in ref. [4]. The full line in Fig. 6 shows the 
equation of state of pure neutron matter obtained in mean-field approximation of the $n n$ contact interaction proportional to $\gamma_{n}+1$ after adjusting $\gamma_{n}=0.788$ to the empirical value of the asymmetry energy $A\left(k_{f 0}=270.3 \mathrm{MeV}\right)=33.2 \mathrm{MeV}$. The downward bending of the full curve in Fig. 6 is weaker and it sets in at somewhat higher densities $\rho_{n}>0.2 \mathrm{fm}^{-3}$. The dotted line in Fig. 6 stems from the many-body calculation of the Urbana group [16]. This curve should be considered as a representative of the host of existing realistic neutron matter calculations which scatter around it. The systematic deviations observed in Fig. 6 indicate that the neutron matter equation of state of ref. [4] cannot be improved by a different treatment of

the short-range NN-dynamics alone. The downward bending of $\bar{E}_{n}\left(k_{n}\right)$ above $\rho_{n}>0.2 \mathrm{fm}^{-3}$ seems to be a generic feature of perturbative chiral $\pi N$-dynamics truncated at three-loop order.

\section{Asymmetry energy}

Finally, we turn to the density dependent asymmetry energy $A\left(k_{f}\right)$ in the approach of ref.[3]. The asymmetry energy is generally defined by the expansion of the energy per particle of isospinasymmetric nuclear matter (described by different proton and neutron Fermi momenta $k_{p, n}=$ $\left.k_{f}(1 \mp \delta)^{1 / 3}\right)$ around the symmetry line: $\bar{E}_{\text {as }}\left(k_{p}, k_{n}\right)=\bar{E}\left(k_{f}\right)+\delta^{2} A\left(k_{f}\right)+\mathcal{O}\left(\delta^{4}\right)$. Evaluation of the first diagram in Fig. 1 leads to the following contribution to the asymmetry energy:

$$
A\left(k_{f}\right)=\frac{g_{A}^{2} k_{f}^{3}}{3\left(4 \pi f_{\pi}\right)^{2}}\left(3 \gamma-2 \gamma_{n}+1\right),
$$

with the coefficients $\gamma=2\left(g_{0}+g_{1}\right) / g_{A}^{2}$ and $\gamma_{n}=4 g_{1} / g_{A}^{2}$ in the notation of ref. [3]. Putting a medium-insertion at each of two nucleon propagators with equal orientation one gets from the second and third diagram in Fig. 1:

$$
\begin{aligned}
A\left(k_{f}\right)= & \frac{g_{A}^{4} M m_{\pi}^{4}}{3(8 \pi)^{3} f_{\pi}^{4}}\left\{2(\gamma+1) u+8\left(2 \gamma-\gamma_{n}+1\right) u^{2} \arctan 2 u\right. \\
& \left.+\left[\left(2 \gamma_{n}-6 \gamma-4\right) u-\frac{\gamma+1}{2 u}\right] \ln \left(1+4 u^{2}\right)\right\}
\end{aligned}
$$

The same diagrams with three medium-insertions give rise to the following contribution to the asymmetry energy:

$$
\begin{aligned}
A\left(k_{f}\right)= & \frac{g_{A}^{4} M m_{\pi}^{4}}{\left(4 \pi f_{\pi}\right)^{4} u^{3}} \int_{0}^{u} d x x^{2} \int_{-1}^{1} d y\left\{\left[2 u x y+\left(u^{2}-x^{2} y^{2}\right) H\right]\left(4 s s^{\prime}-\frac{2}{3} s^{\prime 2}-\frac{2}{3} s s^{\prime \prime}-\frac{7}{2} s^{2}\right)\right. \\
& +(\gamma+1)\left\{\left[\frac{u x y\left(11 u^{2}-15 x^{2} y^{2}\right)}{3\left(u^{2}-x^{2} y^{2}\right)}+\frac{1}{2}\left(u^{2}-5 x^{2} y^{2}\right) H\right] \ln \left(1+s^{2}\right)-\frac{4 u^{2} s^{2} H}{3\left(1+s^{2}\right)}\right. \\
& \left.+\frac{2 u x y+\left(u^{2}-x^{2} y^{2}\right) H}{6\left(1+s^{2}\right)^{2}}\left[8 s\left(1+s^{2}\right)\left(3 s+s^{\prime \prime}-5 s^{\prime}\right)+\left(1-s^{2}\right)\left(3 s^{2}-8 s s^{\prime}+8 s^{\prime 2}\right)\right]\right\} \\
& \left.+2 u^{2}\left(\gamma_{n}+1\right)\left[\frac{2 u x y \ln \left(1+s^{2}\right)}{3\left(u^{2}-x^{2} y^{2}\right)}+\left(\ln \left(1+s^{2}\right)+\frac{2 s^{2}}{3\left(1+s^{2}\right)}\right) H\right]\right\}
\end{aligned}
$$

with $s^{\prime}=u \partial s / \partial u$ and $s^{\prime \prime}=u^{2} \partial^{2} s / \partial u^{2}$ denoting partial derivatives. In the chiral limit $m_{\pi}=0$ only the terms in the first line of eq.(14) survive. The corresponding double integral $\int_{0}^{u} d x x^{2} \int_{-1}^{1} d y \ldots$ has the value $4 u^{7}(\ln 4-1) / 15$. The asymmetry energy is completed by adding to the terms eqs. $(12,13,14)$ the contributions from the kinetic energy, (static) $1 \pi$ exchange and iterated $1 \pi$-exchange written down in eqs.(20-26) of ref.[四. 


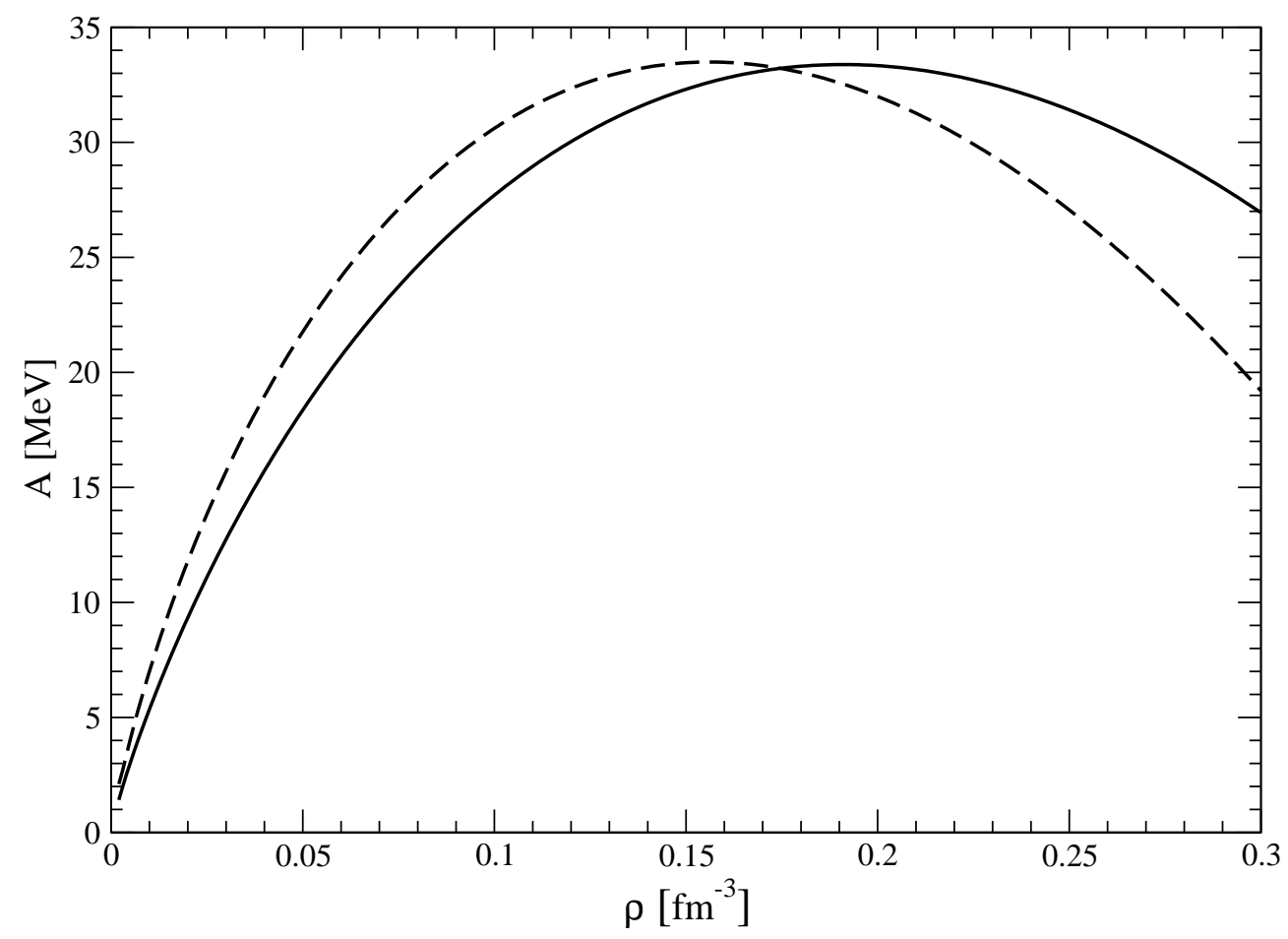

Figure 7: The asymmetry energy $A\left(k_{f}\right)$ versus the nucleon density $\rho=2 k_{f}^{3} / 3 \pi^{2}$. The dashed line corresponds to the approach of Lutz et al. [3] and the full line shows the result obtained in mean-field approximation of the NN-contact interactions. The parameter $\gamma_{n}$ is in each case adjusted to the (empirical) value $A\left(k_{f 0}\right)=33.2 \mathrm{MeV}$ [9].

The dashed line in Fig. 7 shows the density dependence of the asymmetry energy $A\left(k_{f}\right)$ in the approach of Lutz et al. [3] with the coefficient $\gamma_{n}=0.055$ adjusted (at fixed $\gamma=4.086$ ) to the empirical value $A\left(k_{f 0}=250.1 \mathrm{MeV}\right)=33.2 \mathrm{MeV}$ [9]. The full line in Fig. 7 corresponds to the result obtained in mean-field approximation of the NN-contact interaction by dropping the contributions eqs. $(13,14)$. In that case the empirical value $A\left(k_{f_{0}}=270.3 \mathrm{MeV}\right)=33.2 \mathrm{MeV}$ [9] is reproduced by tuning (at fixed $\gamma=6.198$ ) the coefficient $\gamma_{n}$ to the value $\gamma_{n}=0.788$. Both curves in Fig. 7 behave rather similarly. In each case the asymmetry $A\left(k_{f}\right)$ reaches it maximum close to the respective saturation density $\rho_{0}$ and then it starts to bend downward. Since the same (unusual) feature has also been observed in ref.[四] it seems to be generic for perturbative chiral $\pi N$-dynamics truncated at three-loop order.

\section{Concluding remarks}

In this work have we continued and extended the chiral approach to nuclear matter of Lutz et al. [3] by calculating the underlying single-particle potential. The potential for a nucleon at the bottom of the Fermi-sea $U\left(0, k_{f 0}\right)=-20.0 \mathrm{MeV}$ is not deep enough. Most seriously, the total single-particle energy $T_{\text {kin }}(p)+U\left(p, k_{f 0}\right)$ does not grow monotonically with the nucleon momentum $p$. The thereof implied negative effective nucleon mass at the Fermi-surface $M^{*}\left(k_{f 0}\right) \simeq-3.5 M$ and the negative density of states will ruin the behavior of nuclear matter at finite temperatures. The half-width of nucleon-holes at the bottom of the Fermi-sea $W\left(0, k_{f 0}\right)=51.1 \mathrm{MeV}$ comes also out too large in that approach. A good nuclear matter equation of state and better (but still not yet optimal) single-particle properties can be obtained if 
the NN-contact interaction (proportional to the coefficient $g_{0}+g_{1}+g_{A}^{2} / 2$ ) is kept at the meanfield level and not further iterated. The energy per particle of pure neutron matter $\bar{E}_{n}\left(k_{n}\right)$ and the asymmetry energy $A\left(k_{f}\right)$ depend on a second parameter $g_{1}$ in the scheme of ref. [3]. Their density dependence is similar to the results of the one-parameter calculation in ref. 迎. The downward bending of $\bar{E}_{n}\left(k_{n}\right)$ and $A\left(k_{f}\right)$ above saturation density $\rho_{0}$ (less pronounced if the NN-contact interaction is kept at mean-field level) seems to be generic for perturbative chiral $\pi N$-dynamics. More elaborate calculations of nuclear matter in effective (chiral) field theory which fulfill all (semi)-empirical constraints are necessary.

\section{Acknowledgment}

We thank M. Lutz for useful discussions.

\section{References}

[1] A. Akmal, V.R. Pandharipande, D.G. Ravenhall, Phys. Rev. C58 (1998) 1804; and refs. therein.

[2] P. Ring, Prog. Part. Nucl. Phys. 37 (1996) 193; and refs. therein.

[3] M. Lutz, B. Friman and Ch. Appel, Phys. Lett. B474 (2000) 7.

[4] N. Kaiser, S. Fritsch and W. Weise, Nucl. Phys. A697 (2002) 255.

[5] N. Kaiser, S. Fritsch and W. Weise, Nucl. Phys. A700 (2002) 343.

[6] M. Lutz, Nucl. Phys. A677 (2000) 241.

[7] S. Fritsch, N. Kaiser and W. Weise, nucl-th/0202005; submitted to Phys. Lett. B.

[8] M.M. Pavan et al., Physica Scripta T87 (2000) 65.

[9] P.A. Seeger and W.M. Howard, Nucl. Phys. A238 (1975) 491.

[10] P.E. Hodgson, Growth Points in Nuclear Physics, Vol. 3, Pergamon Press, (1981), chapt. 2.

[11] A. Bohr and B.R. Mottelson, Nuclear Structure, Vol.I, Benjamin, (1969), chapt. 2.4.

[12] N.M. Hugenholtz and L. Van Hove, Physica 24 (1958) 363.

[13] P. Grange, J.P. Cugnon and A. Lejeune, Nucl. Phys. A473 (1987) 365.

[14] R.W. Hasse and P. Schuck, Nucl. Phys. A445 (1985) 205.

[15] J.M. Luttinger, Phys. Rev. 121 (1961) 942.

[16] B. Friedman and V.R. Pandharipande, Nucl. Phys. A361 (1981) 502. 